\title{
Welche Unterscheidungen braucht die Endlagerforschung?
}

\section{Soziotechnische Gestaltung zwischen Möglichkeit und Unmöglichkeit}

Andreas Lösch

\section{Zusammenfassung}

Soziotechnische Gestaltung scheint im Fall der nuklearen Entsorgung aufgrund des Sachzwangs vorhandenen Atommülls und geologischer Gegebenheiten von möglichen Endlagerstätten nur schwer oder sogar unmöglich. Denn soziotechnische Gestaltung impliziert die Mitgestaltung von Technik durch die Interaktion unterschiedlichster Gruppen der Gesellschaft - so zum Beispiel Wissenschaft, Politik, Behörden, Zivilgesellschaft. Aus Sicht der Techniksoziologie und der Science $\&$ Technology Studies (STS) ist soziotechnische Gestaltung bzw. die Ko-Produktion von Technik und Sozialem in modernen Gesellschaften aber immer möglich - jedoch nicht unbegrenzt, sondern nur unter spezifischen Voraussetzungen. Der Beitrag führt in grundlegende Theoriemodelle soziotechnischer Gestaltung - soziale Schließungen in der Technikgenese, Gestaltbarkeit soziotechnischer Systeme und die wechselseitige Bedingtheit gesamtgesellschaftlicher Dynamiken, soziotechnischer Regime und innovativer Nischen - ein. Im Horizont dieser Modelle wird diskutiert, unter welchen Bedingungen soziotechnisches Gestalten auch in der nuklearen Entsorgung möglich ist.

\section{Einleitung}

Öffentlichkeitsbeteiligung und Mitgestaltung bei der Entwicklung technischer Infrastrukturen für das nukleare Abfallmanagement waren nicht nur in Deutschland über lange Zeit nicht vorgesehen. ${ }^{1}$ Dies hat sich geändert, wie sich am Fall des neuen Standortauswahlgesetzes für die Entsorgung hochradioaktiver Abfälle erkennen lässt. Die Erwartung relevanter und betroffener gesellschaftlicher Gruppen (so z.B. Wissenschaft, Politik, Behörden, Zivilgesellschaft) bei der Gestaltung von Anlagen zur nuklearen Entsorgung eingebunden zu werden, wird nicht mehr durch betonte Sachzwänge wie zu

1 Der Beitrag entstand auf der Basis einer Keynote, die der Autor auf der SOTEC-radioAbschlusskonferenz hielt, die im Februar 2020 in Berlin stattfand. 
erbringende Entsorgungsnachweise für den Weiterbetrieb von Kernreaktoren und geologische Gegebenheiten für unmöglich gehalten. Vielmehr sollen die Erwartungen auch regulatorisch in ein lernendes und ergebnisoffenes Verfahren für die Errichtung eines geologischen Tiefenlagers eingebunden werden (Smeddinck 2019).

Der Ausschluss von Stimmen aus Protestbewegungen (wie Anti-Atom-Bewegung oder die Bürgerinitiative in Gorleben) von staatlich autorisierter Gestaltung bei der nuklearen Entsorgung wird zumindest bis zu einem gewissen Grad bei der Entscheidungsvorbereitung zurückgenommen. Es sind damit nicht mehr allein staatliche Institutionen, deren Entscheidungen und Verfahren sich früher primär auf natur- und technikwissenschaftliches Wissen gründeten, die heute zu Wort kommen und den Erwartungshorizont mitbestimmen. Dass es dazu kam, wurde insbesondere bei der Entwicklung hin zu dem gegenwärtigen Verfahren der Standortsuche für hochradioaktive Abfälle von bisher weitgehend ausgeschlossenen Akteuren wie insbesondere den nuklearkritischen Protestbewegungen beeinflusst, was auch in der Öffentlichkeit weitgehend akzeptiert wird.

Politisch eingesetzte Gremien, die heute die Governance der nuklearen Entsorgung mit prägen (wie das Nationale Begleitgremium, die Endlagerkommission, beteiligte Wissenschaften und Behörden sowie Vertreter*innen aus Industrie und Zivilgesellschaft) wirken entsprechend nach StandAG bei Umsetzung und Beratungen innerhalb wichtiger Schritte bei der Standortsuche für ein Endlager für hochradioaktive Abfälle mit. Die letztliche Standortwahl basiert also auf politischen Abwägungen, die sich an durchaus konkurrierenden natur- und technikwissenschaftlichen Expertisen orientieren (siehe auch den Beitrag Chaudry/Seidl »Expert*innendissens und das reversible Verfahren der Suche nach einem Endlagerstandort für hochradioaktive Abfälle« in diesem Band). Es zeichnet sich damit eine zunehmende staatlich organisierte Beteiligung der Öffentlichkeit durch Bürger"innen im Suchprozess ab (vgl. z.B. Kallenbach-Herbert et al. 2018: 15). Dennoch bleibt die Frage offen, in welchem Maße unterschiedliche Akteure der Gesellschaft auf welcher Ebene Entsorgung nuklearer Abfälle mitgestalten können. Die hier beschriebenen Überlegungen richten sich vor allem auf die Entsorgung hochradioaktiver Abfälle, sind aber auch auf die nukleare Entsorgung allgemein anzuwenden. Eingedenk dieser Überlegungen wird im Folgenden Gestalten nicht als politische oder gesellschaftliche Aktivität, sondern als soziotechnischer Prozess in den Blick genommen.

Aufgrund der Erkenntnisse aus Techniksoziologie und den Science \& Technology Studies (STS) zu vergangenen und gegenwärtigen Technikentwicklungen in der modernen Gesellschaft ist Gestaltung von Technik auch bei komplexen Systemen zwar immer möglich. Damit dies gelingt müssen aber bestimmte Bedingungen erfüllt sein. Dies entspricht auch der Perspektive der Technikfolgenabschätzung (TA) auf gesellschaftliche Gestaltung von Technik (Grunwald 2002: 37-43). Diese Bedingungen setzen je nach Stadium einer Technikentwicklung oder systemischem Charakter der Technik den Gestaltungsmöglichkeiten Grenzen. Gleichermaßen weisen gerade die Begrenzungen von Gestaltungsoptionen auch auf Möglichkeiten der Einflussnahme durch Intervention, Partizipation und Systemtransformation hin.

Zunächst ist zu betonen, dass aus Perspektive von Techniksoziologie und STS die Analyse von Wechselwirkungen und Ko-Produktionen von Technischem und Sozialem 
grundlegend ist, um überhaupt die moderne Gesellschaft als technisierte Gesellschaft verstehen und untersuchen zu können (Lösch 2012: 252). Gestaltung wird dementsprechend als ko-produktiver Prozess zwischen technischen und sozialen Faktoren gedacht und ist insofern als soziotechnische Gestaltung konzipiert. Unterschiedliche Formen, Funktionen und Prozesse soziotechnischer Gestaltung sind der normale Forschungsgegenstand dieser Forschungsrichtungen (vgl. u.a. Felt et al. 2016; Hackett et al. 2007; Rammert 2000; Weyer et al. 1997). Je nachdem, ob die Entwicklung von Artefakten (z.B. Fahrrad, Telefon), von Prozessen in Produktion und Organisation (z.B. Automatisierung, Digitalisierung in der Industrie) oder von Systemtransformationen (z.B. Elektrifizierung, Energiewende) untersucht werden, zeigen sich unterschiedliche Bedingungen und Möglichkeiten für soziotechnische Gestaltung.

Im Falle der nuklearen Entsorgung waren politisch-öffentliche Diskurse sowie Expertendiskurse seit Jahrzehnten sehr stark von technikdeterministischen Grundannahmen des Sachzwangs geprägt, die soziotechnische Gestaltungen weitgehend ausschlossen (Hocke 2016, siehe auch den Beitrag von Brunnengräber et al. »Der Atommüll eine soziotechnische Tatsache« in diesem Band). Demgegenüber, so die Leitthese dieses Beitrags, lassen sich Bedingungen und Möglichkeiten soziotechnischer Gestaltung auch bei der nuklearen Entsorgung identifizieren. Sie werden erkennbar, wenn die Gestaltbarkeit nuklearer Entsorgung durch die Brille sozialkonstruktivistischer Theoriemodelle der Techniksoziologie und STS betrachtet wird.

Im Folgenden stellt dieser Beitrag unterschiedliche und klassische Theoriemodelle der Techniksoziologie und STS vor, in denen die soziotechnische Gestaltung eine fundamentale Rolle für die Entwicklung von Technik spielt. Dabei werden nicht die neuesten Entwicklungen in Techniksoziologie und STS referiert, sondern Grundmodelle, die für die Auseinandersetzungen dieser Wissenschaftsrichtungen mit soziotechnischer Gestaltung trotz aller Differenzierungen noch immer als Referenz und Orientierungsfolie dienen. Denn der Sinn dieses Vorgehens ist es, der interdisziplinären Forschung zur Entsorgung als soziotechnisches Projekt, heuristische Werkzeuge anzubieten, an denen sich soziotechnische Gestaltungspraktiken möglichst aussichtsreich ${ }^{2}$ orientieren können. Gerade für die interdisziplinäre Forschung ist es wichtig, argumentativ auf Optionen und Grenzen der Gestaltbarkeit aufmerksam zu machen und insofern Hinweise für aussichtsreiche Interventionen zu bieten. Gegenstand und Ziel des Beitrags sind damit nicht die vertiefte empirische Rekonstruktion von Veränderungen soziotechnischer Gestaltungspraktiken und -prozesse im System der nuklearen Entsorgung in Vergangenheit und Gegenwart, sondern die Reflexion der Bedeutung der in den soziologischen Theoriemodellen behandelten Mechanismen und Prozesse soziotechnischer Gestaltung für die Identifikation von Gestaltungsoptionen im System der nuklearen Entsorgung.

Der Einstieg in den Beitrag erfolgt über die Diskussion des Mechanismus der »sozialen Schließung «, wie er in der Forschung zur Technikgenese formuliert wird. Daran schließt die Vorstellung des Modells der Evolution großer "soziotechnischer Systeme« und seiner Implikationen für Systemgestaltungen an. Diesem folgt die Auseinandersetzung mit Dynamiken, die einem Wandel soziotechnischer Systeme entsprechend

2 Aussichtsreich ist hier zu verstehen entsprechend der Intention des Projektes»SOTEC-radio« (vgl. Kallenbach-Herbert et al. 2018). 
des Mehrebenen-Modells der »Transition-Research« vorauszusetzen sind. Das Arrangement der Kapitel folgt dem Prinzip zunehmender Komplexität der vorgestellten Theoriebausteine. Das Verständnis der komplexeren (systemisch-mehrdimensionalen) Modelle baut auf dem der in den einfacheren beziehungsweise eindimensionalen Modellen auf. In allen Kapiteln wird die Vorstellung der Theoriemodelle mit Reflexionen ihrer Bedeutung für die Frage der soziotechnischen Gestaltung im Fall der nuklearen Entsorgung verbunden. Der Beitrag schließt mit Kernaussagen beziehungsweise einigen Merkpunkten für Optionen soziotechnischer Gestaltung der nuklearen Entsorgung, die sich aus der Auseinandersetzung mit den Theoriemodellen ergeben.

\section{Was bedeutet soziotechnische Gestaltung?}

Das mittlerweile klassische sozialkonstruktivistische Modell soziotechnischer Gestaltung in Prozessen der Entwicklung und Durchsetzung einer neuen Technologie ist die »Social Construction of Technology (SCOT)« (Pinch/Bijker 1987). Dieses Modell war und ist für die Techniksoziologie trotz vielseitiger Weiterentwicklungen und Differenzierungen richtungsweisend (z.B. Weyer 2008: 184ff.). Trevor Pinch und Wiebe Bijker entwickelten dieses Modell am historischen Beispiel der Durchsetzung des Sicherheitsfahrrades (1879 bis 1889), dem originären Grundtypus heutiger Fahrräder. Aus ihrem sozialkonstruktivistischen Blickwinkel erklärten sie die Durchsetzung dieses Typs gegenüber anderen Typen ausgehend von Aushandlungsprozessen zwischen sozialen Gruppen. Damit grenzten sie sich von traditionellen Erklärungen der Technikgeschichte $a b$, die die Durchsetzung des einen Typs auf die bessere technische Funktionalität eines technischen Artefakts und ein lineares Innovationsmodell zurückführen (Pinch/Bijker 1987: 32ff.). Ausgangspunkt des Aushandlungsprozesses ist bei ihnen der Konflikt zwischen unterschiedlichen Interessensgruppen von Radsportler*innen und Alltagsradler*innen um die Entwicklung des Fahrrades.

Während Radsportler*innen vor allem an einem technischen Artefakt interessiert sind, das mit möglichst hoher Geschwindigkeit zu fahren sein soll, sind die Alltagsradler*innen vor allem an einem bequemen und vor allem nicht vibrierenden Fahren interessiert. Im Verlaufe des Konflikts kommt es zu einer sozialen Schließung. Soziale Schließung bedeutet, dass der Konflikt zwischen den konträren Positionen der Gruppen dadurch gelöst wird, dass man sich durch Interaktion der mitgestaltenden Akteure auf eine Problemlösung verständigen kann, die für die Interessen beider Gruppen tragfähig ist. Durch diese Einigung wird der Konflikt aufgelöst. Beim Fahrradbeispiel geschieht dies mit der Erfindung der Luftbereifung, die eine Lösung der unterschiedlichen Probleme beider Gruppen ermöglicht. Es kommt zu einer Übersetzung zwischen den konträren Interessen in eine für alle akzeptable Problemlösung. Damit ist der Konflikt beendet und in Folge setzt sich der bis heute bekannte Grundtyp des Sicherheitsfahrrades durch (vgl. Abbildung 1). Voraussetzung dafür sind im Modell von Pinch und Bijker drei sich ergänzende Faktoren: Erstens muss das Artefakt interpretativ flexibel sein; das heißt, es muss sich als Lösung unterschiedlichster Bedürfnisse interpretieren lassen (dies.: 40f.). Zweitens muss es eine Kontroverse zwischen konträren Interessensgruppen geben, sonst kommt es nicht zu einer sozialen Schließung (ebd.: 44f.). Denn ohne Kon- 
Abbildung 1: Schließung durch Übersetzung (SCOT)

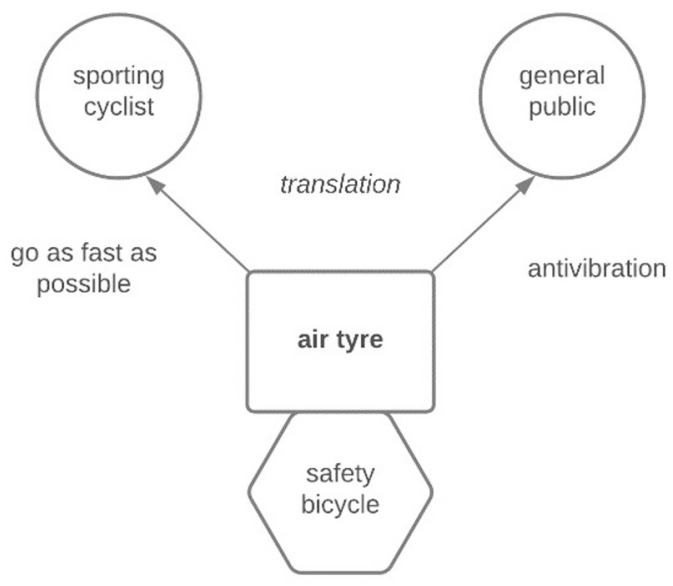

Quelle: Weyer 2008: 183

troverse gäbe es keinen Bedarf an einer Einigung im Sinne einer sozialen Schließung. Drittens muss der Aushandlungseffekt der sozialen Schließung über das luftbereifte Sicherheitsfahrrad in die Weiten der Gesellschaft hineinwirken; es muss also zu einer soziopolitischen Kontextualisierung kommen. Dies geschieht durch die Schaffung entsprechender Infrastrukturen und Regularien (z.B. durch Verkehrswege und -regeln) und die soziokulturelle Normalisierung des Fahrrades als Fortbewegungsmittel unterschiedlichster Gruppen (dies.: 46f.).

Das luftbereifte Fahrrad für sich genommen ist eine technische Konstruktion. Dass sich diese Konstruktion aber gegenüber anderen technischen Konstruktionen durchsetzt, begründet sich über den skizzierten sozialen Aushandlungs- und Kontextualisierungsprozess, weshalb von sozialer Konstruktion der Technik die Rede ist. Weil damit technische und soziale Faktoren sich im Prozess gegenseitig bedingen, handelt es sich um einen Prozess soziotechnischer Gestaltung. Zentral für das Funktionieren soziotechnischer Gestaltung ist in diesem Modell der Mechanismus der sozialen Schließung, denn durch ihn werden Optionen verknappt, was für die Durchsetzung eines Techniktyps und den Gestaltungsprozess eine entscheidende und richtungsgebende Bedingung ist (vgl. auch Weyer 2008: 182-183).

Betrachtet man den Fall der nuklearen Entsorgung durch die Brille des SCOTModells, so stellt sich die Frage, inwieweit eine soziale Schließung als Gestaltungsvorrausetzung bereits stattfindet oder wie eine soziale Schließung in diesem aktuellen Fall möglich wäre. Unbestreitbar finden sich grundlegende Faktoren für eine soziale Schließung entsprechend des SCOT-Modells. Aus dieser Theorieperspektive ist die jahrzehntelang andauernde Kontroverse zwischen Interessensgruppen um Problemdefinitionen 
und Problemlösungen zur nuklearen Entsorgung, die zwischen Staat, Kommunen, Ministerien, Behörden, wissenschaftlichen Expert*innen, Anti-AKW-Bewegung und Anwohner*innen potenzieller Endlagerstätten geführt wird (z.B. Brunnengräber/Di Nucci 2019; Roose 2010), eine Voraussetzung heutiger sozialer Schließungen und damit soziotechnischer Gestaltung der nuklearen Entsorgung. In der Diskussion der letzten Jahre um Optionen nuklearer Entsorgung und die Auswahl von möglichen Standorten finden sich alle Bedingungen für soziale Schließungen wieder. Nukleare Entsorgung lässt sich beispielsweise interpretativ flexibel als wartungsfreie Deponierung radioaktiver Abfälle im tiefen geologischen Untergrund ohne Rückholbarkeit (Endlagerung) oder mit Rückholbarkeit begreifen (Hocke et al. 2016; Kallenbach-Herbert et al. 2018: 55ff.). Es gibt zum Beispiel Expert*innendissense zur Standortauswahl, über die Partizipation zivilgesellschaftlicher Akteure oder überhaupt die Funktion von Partizipation im Verfahren der Standortsuche (siehe auch den Beitrag von Themann »Zum politischen Umgang mit Expert*innendissens « in diesem Band). Offen ist, ob und inwieweit durch die Arbeit der Endlagerkommission (Kommission Lagerung hochradioaktiver Abfallstoffe 2016) oder dem modifizierten StandAG (Kallenbach-Herbert et al. 2018: 14) bereits eine soziale Schließung stattgefunden hat oder sich als Option zumindest abzeichnet. Man kann sagen, dass über die parlamentarisch eingesetzte Endlagerkommission die Kontroverse bereits soziopolitisch kontextualisiert wird. Aber handelt es sich bereits um die Kontextualisierung des Ergebnisses einer Schließung? Bis heute scheint ja offen, wie es zu Übersetzungen und Re-Definitionen von Problemlösungen kommt, die nach dem Modell als Bedingung von sozialer Schließung zwischen den konträren Standpunkten der betroffenen Akteursgruppen angesehen werden. Könnte dies die Problemlösung eines Standortauswahlverfahrens mit Rückholbarkeit und Reversibilität im Tiefenlager sein? Im Gegensatz einer frühen und einmaligen sozialen Schließung im Fahrradbeispiel nach dem SCOT-Modell scheint man hier eher mit einer Serie von mehreren sozialen Schließungen konfrontiert. Jede soziale Schließung wäre dann eine gestaltende Intervention, die den Entwicklungsverlauf der nuklearen Entsorgung beeinflusst.

Doch wie zukunfts-, gestaltungsoffen und auch reversibel sind soziale Schließungen in soziotechnischen Prozessverläufen? Hierzu gibt das Konzept der »Technikgenese« Auskunft (Rammert 2000; Weyer 2008; Weyer et al. 1997). Immer wiederkehrende soziale Schließungen in unterschiedlichen Phasen der Technikentwicklung hat die Techniksoziologie vor einigen Jahren an ganz unterschiedlichen Beispielen (z.B. Airbus, Transrapid, Personal Computer, Satellitenfernsehen) untersucht. In diesem Modell geht es zum einen um das Verstehen des Zusammenhangs sozialer Schließungen und die Entstehung von Pfadabhängigkeiten in Technikentwicklungsprozessen. Pfadabhängigkeiten minimieren die Möglichkeit gestaltender Intervention. Wie das Schaubild aber zeigt (Abbildung 2), kommt es im Zeitverlauf eines Prozesses immer wieder zu Verzweigungen und sozialen Schließungen, die eingeschlagene Pfade auch wieder aufbrechen können. An den Verzweigungspunkten kommen veränderte oder neue Akteurskonstellationen ins Spiel. An diesen Punkten können Akteure »die Entwicklung des Pfades aktiv gestalten «, Sachzwänge durchbrechen und alternative Handlungsoptionen stärken oder gar durchsetzen. »[A]ber sie tun dies innerhalb des gegebenen Rahmens 
technischer, sozialer und politischer Optionen, die ihrerseits (pfadabhängiges) Resultat vorheriger Entscheidungen und Handlungen sind« (Weyer 2008: 188).

Abbildung 2: Phasen der Technikgenese

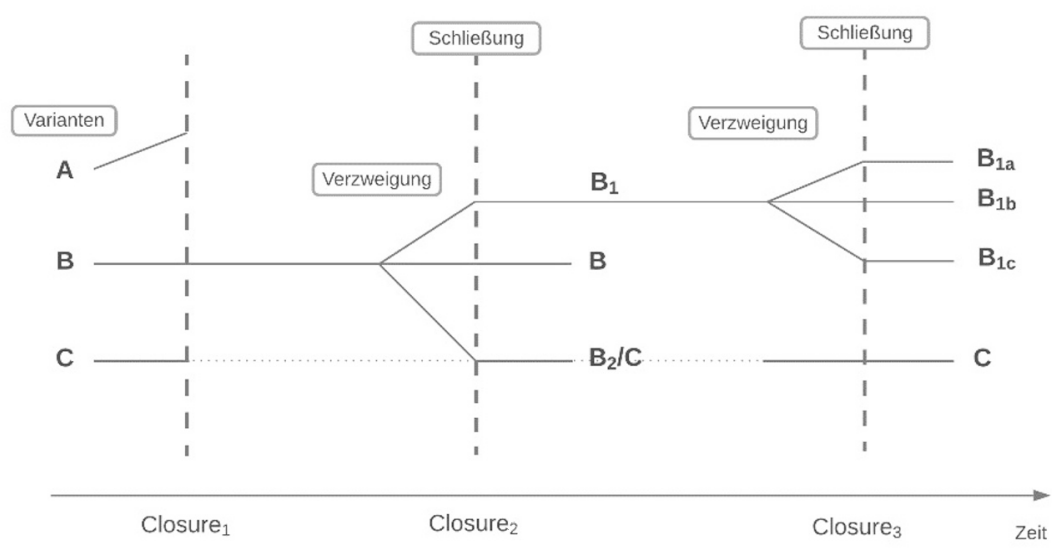

Quelle: Weyer 2008: 187

Eine Schließung in einer Frühphase (wie im SCOT-Modell) determiniert also nicht den gesamten Prozessverlauf. Sie schränkt aber Variationsmöglichkeiten ein; gleichermaßen ist diese Einschränkung auch eine richtungsgebende Voraussetzung dafür, dass überhaupt ein entsprechender Prozess in Gang kommt. Zu bestimmten Zeitpunkten können neue Varianten an Problemlösungen ins Spiel kommen und den Prozess gestaltend beeinflussen. Wesentliche Rahmenbedingung für die Technikentwicklung, aber eben auch für Gestaltungen im Verlauf des Prozesses ist die »Generierung eines soziotechnischen Kerns (Weyer 2008: 190) bereits in der Frühphase einer Entwicklung. Ein solcher Kern besteht nach Weyer »aus einer technisch-instrumentellen Konfiguration (in Form eines allgemeinen Konstruktionsprinzips)« sowie einer »sozialen Konfiguration (in Form eines antizipierten Arrangements von Akteuren)« (ebd.). Dieser Kern bleibt über den ganzen Prozess erhalten und »stellt ein allgemeines Orientierungsmuster für die Such- und Problemlösungsstrategien« (ebd.) der beteiligten Akteure dar. Der Kern beeinflusst ihre Entscheidungen und Selektionen von Alternativoptionen in den sich wandelnden Akteurskonstellationen in den Phasen der Stabilisierung (etwa die Entwicklung eines Prototyps durch ein enges Akteursnetzwerk) und der Durchsetzung einer technischen Innovation (mittels Dekontextualisierung in einem sich ausweitenden Akteursnetzwerk). In jeder Phase finden soziale Schließungen innerhalb der Akteursnetzwerke statt, die den weiteren Verlauf der Entwicklung beeinflussen (vgl. auch Weyer et al. 1997: 35-53).

Das skizzierte Konzept der Technikgeneseforschung wurde für die Erforschung von Prozessen entwickelt, aus denen wegweisende technische Innovationen hervorgehen können. Die soziotechnische Entsorgungsforschung ist mit einem Prozess 
konfrontiert, welchem gemeinhin eine starke Pfadabhängigkeit zugesprochen wird. Dies geschieht mit der Argumentation, dass aufgrund einer schon vor Jahrzehnten etablierten technisch-instrumentellen Konfiguration (z.B. Bergwerk, Castoren) und dafür seit langem zuständigen sozialen Konfigurationen (Netzwerke aus Ingenieur*innen, Geolog*innen, Behörden) stabile Sachzwänge und Strukturen institutionalisierter Problembearbeitung geschaffen wurden. Doch auch wenn hier vieles stabilisiert und unveränderlich erscheint, finden sich in Geschichte und Gegenwart des Entsorgungsprozesses immer wieder soziale Schließungen und Öffnungen sowie nachfolgende Schließungen als Bedingung und Verknappung von Gestaltungsoptionen. Beispiele hierfür sind die Neuordnungen durch das StandAG, der Bedeutungswandel der Technik und des Wissens, der zunehmend reflektierte Umgang mit Pfadabhängigkeiten als Folge früherer politischer Entscheidungen und im Prinzip auch das Aufkommen einer soziotechnischen Endlagerforschung selbst, die Elemente der Interdisziplinarität stärkt. Als neuer Akteur verändert nämlich die soziotechnische Endlagerforschung die Akteursnetze, die sich mit den Optionen der Endlagerforschung befassen (KallenbachHerbert et al. 2018: 15).

Um Gestaltungsoptionen ausfindig zu machen, ist in den Blick zu nehmen, welche Rahmenbedingungen aber auch Veränderungsoptionen ausgehend vom relativ stabilen soziotechnischen Kern der Entsorgung möglich sind. Einerseits existiert ein stabiler soziotechnischer Kern an grundlegend notwendigen Geräten, Anlagen, Spezialist*innen, Orten (wie Bergwerke) und Verfahrensabläufen. Ins Akteursnetz wurden aber zum Beispiel neue Fachpolitiken, partizipative Elemente und der Einbezug neuer Gruppen integriert. Das gestaltende Akteursnetz und seine Randbedingungen verändern sich also. $\mathrm{Zu}$ diesem neuen Akteursnetz gehören auch ursprünglich externe Gegenexperten. ${ }^{3} \mathrm{Im}$ Prozessverlauf sind also trotz stabilem Kern immer wieder neue soziale Schließungen möglich (z.B. durch neue Akteure, neue politische Beschlüsse, neue geologische Befunde, technische Innovationen u.v.m.). Dies ist gerade eine Voraussetzung für die Entsorgung als »lernendes System « (ebd.: 71ff.), wie dies zum Beispiel im aktuellen Standortauswahlverfahren für hochradioaktive Abfälle mit der Option der Rückholbarkeit und allgemeiner Reversibilität von Entscheidungen konzeptionell gesetzt wurde.

Mit dem Modell der Technikgenese lassen sich also in der Abfolge der Öffnung vergangener sozialer Schließungen und diesen Öffnungen folgenden neuen sozialen Schließungen soziotechnische Gestaltungen im Prozess erkennen. Aufgrund des eindimensionalen Blicks, die die Linearität von Prozessen betont, ist der Erkenntnisbereich des Konzepts Technikgenese in der Sache aber begrenzt, wenn man Interventionsoptionen in komplexen systemischen Zusammenhängen erkennen und verstehen will. Gerade bei der nuklearen Entsorgung geht es nicht nur um Prozessgestaltung, sondern um die Frage der Gestaltbarkeit eines hochkomplexen und sich evolutiv fortentwickelnden soziotechnischen Systems. 


\section{Gestaltung soziotechnischer Systeme im Kontext technologischer Entwicklung}

Die systemische Herausforderung bei der soziotechnischen Gestaltung komplexer funktionaler Zusammenhänge wie der Entsorgung hoch radioaktiver Abfälle wird deutlich, wenn man sich das Theoriekonzept »soziotechnischer Systeme« vor Augen führt. Das in den STS und der Techniksoziologie referierte und bis heute heuristisch eingesetzte Grundmodell soziotechnischer Systeme geht auf die Studie des Historikers Thomas Hughes »Networks of Power« zur Elektrifizierung der westlichen Gesellschaft zwischen 1880 und 1930 zurück (Hughes 1983). An diesem Beispiel beschreibt Hughes die Evolution und Gestaltung "großer technologischer Systeme« (Hughes 1987). Diese Systeme werden in diesem Beitrag aufgrund ihrer sozialen und technischen Komponenten sowie deren Wechselwirkungen als soziotechnische Systeme bezeichnet. Ein solches System ist nach Hughes (1986) einerseits ein nahtloses Netz aus unterschiedlichsten technischen und nicht-technischen Komponenten - wie zum Beispiel technische Artefakte, Organisationen, oder wissenschaftliche Forschung. Andererseits zeichnet sich ein soziotechnisches System durch die Funktionalität eines zielorientierten Gesamtzusammenhangs aus. Die Interaktionen beziehungsweise das Zusammenspiel aller Komponenten des Netzes sind auf die Erreichung des Systemziels hin ausgerichtet und werden durch seine Governance gesteuert.

Beim Elektrizitätssystem in der Studie von Hughes besteht das nahtlose Netz aus technischen Artefakten, organisatorischen Promotoren, technischen Infrastrukturen wie Bergwerken zur Rohstoffgewinnung, wissenschaftlichen und legislativen Komponenten, dem Zugriff auf relativ unbegrenzte natürliche Ressourcen und Entwicklungslabore sowie einem investitionsbereiten Finanzsektor (Hughes 1986: 287). Dieses eng kooperierende Netzwerk wird von Autoren wie Wiebe E. Bijker als soziotechnisches Ensemble (Bijker 1995) verstanden. Dieses Ensemble ist ein Kooperationsnetzwerk mit einem gemeinsamen Ziel und einem für das jeweilige Ensemble spezifischen Governancemodus, der die Integration und Kontrolle systeminterner Prozesse untereinander in Abstimmung mit externen Bedingungen und Prozessen der Umwelten des Systems gewährleisten soll. Die Funktionalität des zielorientierten Gesamtzusammenhangs hängt davon ab, dass einerseits das soziotechnische Ensemble mit seinem Netzwerk zusammengehalten wird und wichtige Akteure (z.B. aus der Grundlagenforschung oder aus dem Finanzsektor) das nahtlose Netz nicht verlassen.

»These components make up a system, because they fall under a central control and interact functionally to fulfill a system goal, or to contribute to a system output. Obviously, an electric-light and power system would not function if all generators were removed; less obviously, the system would also break down, if an investment bank providing funds withdrew from the system « (Hughes 1986: 287).

Andererseits müssen die aufeinander aufbauenden Planungs- und Gestaltungsprozesse des Gesamtsystems mit seinen Umwelten in einem korrespondierenden Verhältnis stehen. Die Elemente und Prozesse des Ensembles und seiner Umwelten müssen in einem synchronen Modus zusammenspielen, ohne dabei trotz gemeinsamer Problemlösungsorientierung ihre Unabhängigkeit und Flexibilität aufgrund von im Prozess entstehen- 
den technologischen Pfadabhängigkeiten zu verlieren (vgl. Hughes 1987: 53). Hierfür ist ein genaueres Verständnis der Governance (bzw. Steuerung und Kontrolle) des Ensembles sowie systemrelevanter externe Umweltbedingungen notwendig.

In Hughes Studie wird die Governance des funktionalen Zusammenspiels durch die System Builders (Hughes 1987) praktiziert. ${ }^{4}$ System Builders sehen sich immer vor große Herausforderungen gestellt. Das System entwickelt, stabilisiert und verbreitet sich nur, wenn es den System Builders gelingt, Erfindungen in geeigneter Form zu kombinieren und Kooperationen mit allen systemrelevanten Institutionen und Organisationen herzustellen. Zudem müssen sie permanent Innovationen hervorbringen, die die reverse salients (Hughes 1987: 73) als die Systemevolution hemmende Faktoren überwinden. Dies können für das System dysfunktionale Organisationen sein, veraltete oder inkompatible Techniken, unpassende eingespielte Verfahrensabläufe oder auch hemmende Regularien (wie nicht-korrespondierende Normen, Standards, Gesetze) innerhalb des Systems sowie in den Systemumwelten, auf die das Funktionieren des Systems angewiesen ist. Das System der jeweiligen Technologie ist zwar von seinen Umwelten abgegrenzt, aber es prozessiert nicht isoliert als geschlossenes System, sondern es entwickelt sich und wird gestaltet innerhalb von "Systemumwelten«, die einerseits das System beeinflussen und andererseits im Zuge seiner Evolution vom System beeinflusst werden. Dabei spielen Schlüsselpersonen ebenso eine Rolle wie Akteursnetze und Organisationen mit ihren zielorientierenden soziotechnischen Kernen (vgl. Abschnitt 2 in diesem Beitrag). Einflussfaktoren sind sowohl ökonomische Aspekte als auch Beharrungskräfte (reverse salients) im System.

Gerade mit dem Etablieren neuer technischer Infrastrukturen gewinnen Beharrungskräfte und gegenläufige Tendenzen an den Schnittstellen der Systembereiche und zu Systemumwelten an Bedeutung. Deren Überwindung und Koordination erfordert fortlaufend Unterstützung durch beteiligte Organisationen und Akteursgruppen, um zu jedem Zeitpunkt das Momentum (beständige Funktionalität und Lernfähigkeit) des Systems zu gewährleisten.

»As the system grew, other kinds of problem developed, some of which can be labeled as >reverse salients‘. [...] As technological systems expand, reverse salients develop. Reverse salients are components in the system that have fallen behind or are out of phase with the others. [...]. Technological systems, even after prolonged growth and consolidation, do not become autonomous, they acquire momentum. [...] The large mass of a technological system arises especially from the organizations and people committed by various interests to the system. Manufacturing corporations, public and private utilities, industrial and government research laboratories, investment and banking houses, sections of technical and scientific societies, departments in educational institutions, and regulatory bodies add greatly to the momentum of modern electric light and power systems« (Hughes 1987: 73; 76f.). 
Für das Verständnis der Systementwicklung und der Gestaltungsmöglichkeiten des Entsorgungssystems durch Akteure impliziert dieses Theoriemodell folgendes: Der Zusammenhang der Entsorgung muss ebenfalls als ein soziotechnisches Ensemble im Sinne eines nahtlosen Netzes aus technischen Anlagen, kollektiven Akteuren, Verfahren, Regularien und entsprechend diverser soziotechnischer Kopplungen und Interaktionen betrachtet werden, die Teil des Systems oder Teil seiner Umwelten sind (KallenbachHerbert et al. 2018: 10). Gleichzeitig ist es ein funktionaler Systemzusammenhang, dessen Komponenten auf die Lösung des Problems der Entsorgung des Atommülls ausgerichtet sind. Entsprechend verändert das Ersetzen von Komponenten oder das NichtFunktionieren nur einer Komponente den Gesamtzusammenhang. Dies stellt diverse Herausforderungen an die Akteure, die die Rolle der System Builders im System innehaben und in dieser Rolle das System gestalten können.

Die gegenwärtigen Neuordnungen des Systems zum Beispiel durch das StandAG erfordern von ihnen die Integration neuer Akteure und Wissensformen (KallenbachHerbert et al. 2018: 15ff.). Diese sind mit dem etablierten und bestehenden Ensemble des Systems zu kombinieren. Reverse salients (wie z.B. hemmende Behördenstrukturen oder nicht mehr passende Grenzwerte) müssen überwunden werden. Das System muss weiterhin fortlaufend an seine Umwelt (z.B. umgebende Infrastrukturen) angepasst werden und diese antizipieren. Dazu gehört auch, unvorhersehbare politische Dynamiken und Veränderungen als Möglichkeit im Blick zu haben. Die Interaktionen von Akteuren und Organisationen innerhalb und außerhalb des Systems müssen in der Terminologie von Hughes ein Momentum, also eine ausreichende Durchschlagskraft und Reflektiertheit gewährleisten. Nur wenn diese Interaktionen nach der Integration neuer Akteure und Organisationen, wie nach der gegenwärtigen partizipativen Öffnung des Entsorgungssystems vorgesehen, gegeben ist, wird die dauerhafte Ermöglichung und Förderung von Lernprozessen des Systems als lernendes System entsprechend der Zielsetzungen der Endlagerkommission zu erwarten sein. ${ }^{5}$ Kurz: Aus einem von wenigen Akteuren und Organisationen gestalteten System muss ein lernendes System werden, an dem viele neue Akteure und Organisationen beteiligt sind. Die offene Frage ist, in welchen Rollen die Beteiligung der neuen Akteure und Organisationen möglich ist. Werden sie selbst Systemgestalter? Oder können sie als ermöglichende Akteure der Umwelt wünschenswerte Systemgestaltungen unterstützen und somit zu einer Realisierung des Systems als (selbst-)lernendes System (Kallenbach-Herbert et al. 2018: 22; 64) beitragen.

Diese systemisch bedingten Herausforderungen an soziotechnische Gestaltung sind schon immens. Ihre Umsetzbarkeit setzt aber miteinander interagierende und korrelierende Dynamiken der Innovation, des Regime- und des Gesellschaftswandels voraus. Dieser Zusammenhang lässt sich mittels der Mehrebenen-Perspektive der Transition-Forschung begreifen. 


\section{Dimensionen soziotechnischer Gestaltung}

Soziotechnische Gestaltung komplexer systemischer Zusammenhänge setzt Transformationen auf unterschiedlichen gesellschaftlichen Ebenen voraus. Diese beeinflussen, dass ein Wandel eines soziotechnischen Systems überhaupt möglich ist. Eine Heuristik zur Identifizierung von Systemwandel und insbesondere von Möglichkeiten der Intervention in ein etabliertes System, um Veränderungen im System zu erreichen, bietet das Mehrebenen-Modell der Transition Research an (z.B. Geels 2002). Nach diesem Modell, an welchem sich mittlerweile unterschiedlichste Fallstudien insbesondere im Bereich der Transformation des Energiesystems oder der Nachhaltigkeitsforschung orientieren (z.B. Büscher/Schippl 2013; Roggema et al. 2012), ist eine Systemtransformation nur möglich, wenn sich auch die etablierten soziotechnischen Regime verändern. Soziotechnische Regime sind mehr als technische Regime. Sie sind zugleich Regelwerke, Heuristiken und Skills als auch heterogene Akteursgruppen.

»A technological regime is the rule-set or grammar embedded in a complex of engineering practices, production process technologies, product characteristics, skills and procedures, ways of handling relevant artefacts and persons, ways of defining problems; all of them embedded in institutions and infrastructures« (Rip/Kemp 1998: 340).

Ähnlich argumentieren auch Frank Geels und René Kemp:

»The regime concept has been further widened to ssocio-technical regimes`, which include scientists, users, policy makers and societal groups besides engineers and firms. These social groups interact and form networks with mutual dependencies, resulting in the alignment of activities. This inter-group coordination is represented with the concept of socio-technical regimes. «(Geels/Kemp 2007: 443)

Solche Regime gewährleisten die Stabilität und das routinemäßige Funktionieren der Systeme (ebd.: 443). Damit sich ein Regime, situiert auf der Mesoebene, überhaupt wandelt, muss nach dem Mehr-Ebenen-Modell eine Wechselwirkung oder mindestens eine Korrelation zwischen Dynamiken auf der Makroebene der Gesellschaft (Landscape) und von Innovationen in Nischen auf der Mikroebene stattfinden. Alle drei Ebenen beeinflussen sich gegenseitig (vgl. Abbildung 3). Nischen sind dabei geschützte experimentelle Räume für Innovationen, die das Regime zulässt, die aber ohne Wandel auf der Makroebene und in Folge auch der Regime selbst, nicht aus ihrer randständigen Nische herauskommen.

Die Vertreter der Transition Research beschreiben die Interaktion der Ebenen und die Möglichkeit des Wandels folgendermaßen:

»Niches may be viewed as a micro-level phenomenon, interacting with the established regimes at the mesolevel, within a macro-landscape. The macro-level is formed by the socio-technical landscape, which refers to aspects of the exogenous environment that is beyond the direct influence of actors. The content of the sociotechnical landscape is heterogeneous and may include aspects such as economic growth, broad political coalitions, cultural and normative values, environmental problems and resource scarcities« (Geels/Kemp 2007: 443). 
Abbildung 3: Mehrebenenmodell der Transition Research

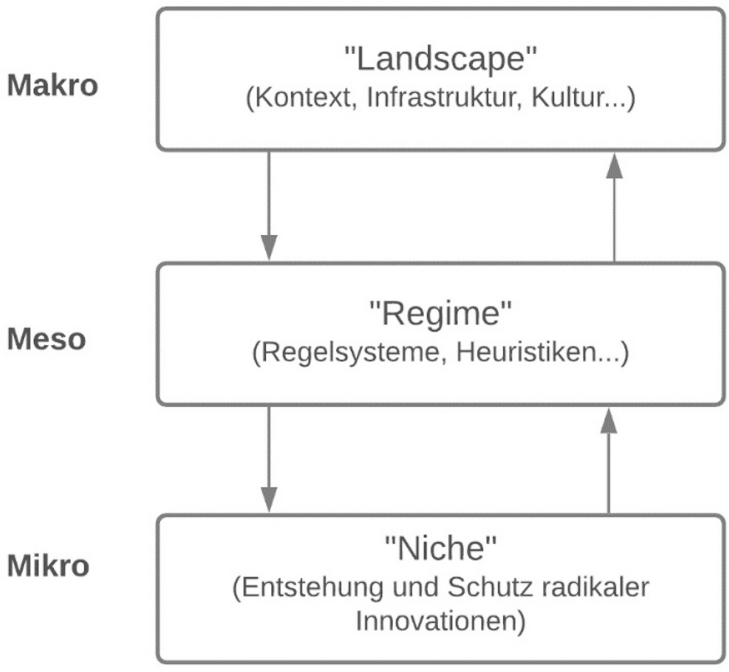

Quelle: Weyer 2008: 198

Breite politische Koalitionen spielen somit ebenso eine Rolle wie kulturelle Phänomene und materielle Ressourcen.

»The key point of the multi-level perspective (MLP) is that system innovations come about through the interplay between processes at different levels in different phases. In the first phase, radical innovations emerge in niches, often outside or on the fringe of the existing regime. There are no stable rules (e.g. dominant design), and actors improvise, and engage in experiments to work out the best design and find out what users want. The networks that carry and support the innovation are small and precarious. The innovations do not (yet) form a threat to the existing regime. In the second phase, the new innovation is used in small market niches, which provide resources for technical development and specialisation. The new technology develops a technical trajectory of its own and rules begin to stabilise (e.g. a dominant design). But the innovation still forms no major threat to the regime, because it is used in specialised market niches. New technologies may remain stuck in these niches for a long time (decades), when they face a mismatch with the existing regime and landscape. The third phase is characterised by wider breakthrough of the new technology and competition with established regime, followed by a stabilisation and new types of structuring (Ceels/Kemp 2007: 443f.).

Nischen sind damit der Ausgangspunkt für systemische Innovationen, für deren Erfolg in der folgenden Phase der Zugriff auf ausreichende Ressourcen ebenso eine Rolle spielt wie die Auseinandersetzung mit konkurrierenden Anwendungen konkreter, 
bereits vorhandener Technologien. Solche Innovationen der Nische waren im Vorfeld der beginnenden Energiewende zum Beispiel erneuerbare Energien (von Windkraft bis Solarenergie), neue Informations- und Kommunikationstechniken (Smart GridTechnologien, Smart Meter), aber auch Innovationen im Verhältnis von Stromkonsum und Stromproduktion (z.B. Kund"innen als Prosumer). Dass diese Innovationen überhaupt einen Einfluss auf den gegenwärtigen Wandel des Energiesystems auf Regimeebene haben können, setzt parallele Dynamiken auf der Ebene der Landscapes, eben des Kontexts des Systems, der umgebenden Infrastrukturen, Politik, Kultur etc. voraus; so zum Beispiel den Prozess der Energiewende, die zunehmende Durchsetzung des Leitbildes der Nachhaltigkeit und auch einen kulturellen Einstellungswandel in Bezug auf Energienutzung (vgl. auch Lösch/Schneider 2016). ${ }^{6}$

Gestaltung war in diesen Beispielen und ist generell bei soziotechnischen Systemen wie der Energie, Mobilität und Digitalisierung nur in Kooperation von Organisationen, Kombination von Techniken und der Modifikation von Regularien möglich, die alle drei Ebenen betreffen und miteinander verbinden. Wenn dies gelingt, ist ein Regimewandel und in Folge Systemwandel möglich. Etablierte Regime sind dabei - im Sinne des Modells soziotechnischer Systeme (vgl. Abschnitt 3 dieses Beitrags) - reverse salients, die das System in seinem Ist-Zustand stabilisieren (Hughes 1987: 73), damit aber seinen Wandel verhindern. Regime sind insofern Behinderungen und gleichermaßen, wenn sie sich verändern, Bedingungen des Wandels.

Betrachtet man Prozesse soziotechnischer Ko-Evolution und Ko-Produktion aus der Sicht des Mehrebenen-Modells, so werden die Optionen einer Gestaltung der Systemtransformation deutlich (vgl. Abbildung 4). Entsprechend der Grafik wird durch Dynamiken auf der Landscape-Ebene ein window of opportunity geschaffen, was einerseits die Innovationen der Nische beeinflusst, andererseits die Regime selbst, die dann gegebenenfalls Nischenexperimente fördern. Im weiteren Verlauf werden Lernprozesse und Neuerungen aus Nischenaktivitäten unterstützt durch unterschiedliche Akteure aus der Gesellschaft. Bestimmte innovative Elemente (z.B. Designs) werden vereinheitlicht und es setzt sich ein neues und dominierendes Design durch. Es kann dann zu Durchbrüchen kommen, die das Regime neu konfigurieren und dieses umstellen oder anpassen. Wenn dies gelingt, beeinflusst ein neues beziehungsweise gewandeltes Regime auch wieder die Landscape und ein neues soziotechnisches System, angepasst an seine Umwelten, ist etabliert oder ein bestehendes System transformiert. ob das gesamte Energiesystem oder das nukleare Entsorgungssystem, ob das ganze Mobilitätssystem oder das System der Automobilität in den Blick genommen wird. Unabhängig davon ist festzuhalten, dass das sozio-technische Regime, in SOTEC-radio als technologisches Regime bezeichnet, in Umwelten eingebunden ist, die nicht eng, sondern vielmehr lose mit der technischen Infrastruktur zum Beispiel zur nuklearen Versorgung verknüpft sind. Straßenverbindungen gehören wegen ihrer Multifunktionalität zwar zur Landscape, nicht aber zum Regime im Vergleich zum Beispiel zu einschlägigen Expertisen oder akademischer Grundlagenforschung zu Nuklear.

7 Die von Roggema et al. erstellte Grafik kombiniert Grafiken aus drei Publikationen von Geels (Geels 2002; ders. 2005 und ders. 2011). 
Abbildung 4: Interaktion zwischen den Ebenen des Mehrebenenmodells

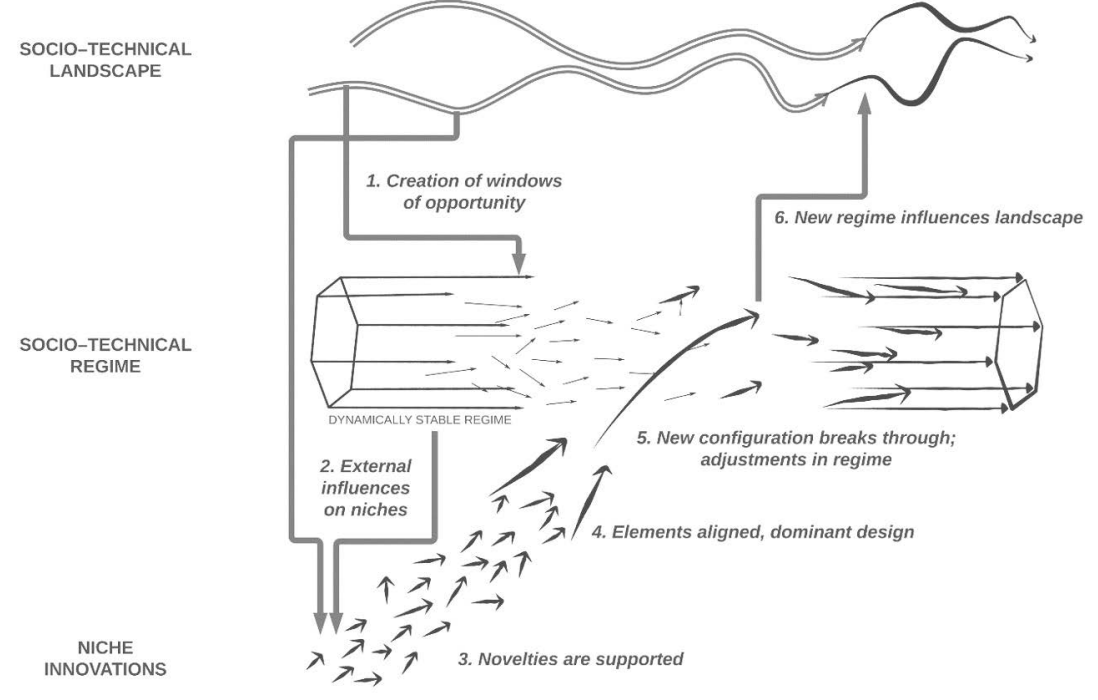

Quelle: Roggema et al. 2012: $2532^{7}$

Durch die Brille dieses Modells lässt sich zum Beispiel auch präziser fragen, wo und wann das System der Entsorgung hoch radioaktiver Abfälle wie umgestaltet wird und noch umgestaltet werden könnte. Ist durch fortschreitende einfache Inklusion regimeexterner Akteure (z.B. im Standortauswahlverfahren) das Entsorgungsregime bereits in Transformation oder transformiert? Haben die, nach dem Modell, bisher externen Nischenaktivitäten des Protests mittlerweile so viel Unterstützung erhalten, dass sie Teil einer Neukonfiguration des Regimes geworden sind? Hat die Endlagerkommission und das modifizierte StandAG, die sich als Effekte eines Wandels der Landscape seit dem Energiewendebeschluss (BMU/BMWI 2011) und partizipativem Wandel der Politik interpretieren lassen, ein Möglichkeitsfenster (window of opportunity) geöffnet - ein Möglichkeitsfenster mit Folgen für Nischen- und Regimewandel? Zeichnet sich durch die Integration partizipativer Elemente ein Wandel auf Regimeebene ab?

\section{Schluss - einige Merkpunkte}

Der Durchgang durch die grundlegenden Modelle der Techniksoziologie und STS macht deutlich, dass soziotechnisches Gestalten immer stattfindet und immer möglich ist. Es zeigt sich aber auch, dass die Gestaltung insbesondere großer soziotechnischer Systeme nicht unbegrenzt, zu jeder Zeit und von allen gesellschaftlichen Gruppen möglich ist. Denn Gestaltung als soziotechnischer Prozess verstanden, setzt die gelunge- 
ne Interaktion und Kombination sozialer und technischer Faktoren voraus. Vielmehr kann Gestalten nur unter spezifischen Voraussetzungen gelingen. Die Theoriemodelle als Heuristiken genutzt, helfen aber auch Interventionspunkte der Gestaltung zu identifizieren: Ausgangslagen, Orte, Zeitpunkte etc.

Daraus ergeben sich für die soziotechnische Gestaltung komplexer Systeme wie der Endlagerung hochradioaktiver Abfälle vor dem Hintergrund der Theoriemodelle folgende Merkpunkte:

1. Soziale Schließungen sind nicht eine Verhinderung von soziotechnischen Gestaltungen. Sie sind trotz der ihnen folgenden Reduktion des Möglichkeitsraumes gerade auch eine Gestaltungsbedingung. Soziale Schließungen setzen unter anderem Kontroversen und Re-Definitionen von Problemlösungen voraus. Beides hat in der Entsorgung hoch radioaktiver Abfälle stattgefunden und begleitet sie möglicherweise fortlaufend.

2. Schließungen in Prozessverläufen sind notwendig, um eine kollektive Zielorientierung zu entwickeln. Gleichzeitig sind sie auch riskant, da sie zu Pfadabhängigkeiten, sowie Einschreibungen der Schließung in Technik oder Infrastrukturen führen können. Trotz der fortlaufenden Möglichkeit von Öffnungen, Variationen und neuen Schließungen stellt sich die Frage nach dem Verhältnis von Reversibilität und Irreversibilität - etwa bei einer Festlegung auf Nichtrückholbarkeit.

3. Der systemische Blick auf Entsorgung als soziotechnisches System muss einerseits das nahtlose Netz aller relevanten technischen und nicht technischen Elemente erfassen, aus dem sich das System zusammensetzt. Zugleich muss dieses Netzwerk als funktionaler Gesamtzusammenhang mit Problemlösungsorientierung aller Elemente betrachtet werden. Nur so werden die Wechselwirkungen und gegenseitigen Bedingtheiten der Elemente erfassbar. Dies ist die Bedingung zur Identifizierung des geeigneten Umgangs mit reverse salients, für Umweltanpassung und Antizipation von Umweltdynamiken des Systems sowie die Ermöglichung von dauerhafter Lernfähigkeit (bzw. die Gewährleistung von Momentum).

4. Der Mehrebenen-Blick zeigt, dass soziotechnisches Gestalten nur als KoGestaltungen zwischen innovativen Aktivitäten in Nischen und gesellschaftlichem Wandel auf der Landscape-Ebene möglich ist. Ohne dieses Zusammenwirken kommt es zu keiner Öffnung und Neukonfiguration der etablierten Regime. Regimetransformation aber ist die Bedingung der Systemtransformation.

Wird Gestaltung als soziotechnischer Prozess wechselseitiger Ko-Konstruktion von Technik und Sozialem begriffen, so öffnet dies den Blick für aussichtsreiche Interventionen und Gestaltungen eines soziotechnischen Systems, in dem - wie im Fall der nuklearen Entsorgung - viele technische Bedingungen nur bedingt variabel sind. Gerade hier ist eine gelungene, das heißt für das System funktionale Abstimmung zwischen gesellschaftlichen Gruppen und Akteuren, politischen Institutionen und technischen Vorgängen und Möglichkeiten notwendig, damit soziale und politische Gestaltungsmaßnahmen nicht ins Leere laufen. Dies ist jedoch mit weiteren empirischen Studien zu untersuchen. Insbesondere für die nukleare Entsorgung, aber auch 
für den gesamten Sektor der Atomenergie fehlen dazu bisher noch einschlägige interund transdisziplinäre Arbeiten.

\section{Literatur}

Bijker, Wiebe. E. (1995): Of Bicycles, Bakelites and Bulbs. Toward a Theory of Sociotechnical Change, Cambridge, Massachusetts: MIT Press.

Brunnengräber, Achim/Di Nucci, Maria Rosaria (Hg.) (2019): Conflicts, Participation and Acceptability in Nuclear Waste Governance. An international Comparison, Vol. III, Wiesbaden: Springer VS.

Bundesministerium für Umwelt, Naturschutz und Reaktorsicherheit (BMU)/Bundeministerium für Wirtschaft und Technologie (BMWI) (Hg.) (2011): Das Energiekonzept der Bundesregierung 2010 und die Energiewende 2011, München: Bundesministerium für Wirtschaft und Energie.

Büscher, Christian/Schippl, Jens (2013): Die Transformation der Energieversorgung: Einheit und Differenz soziotechnischer Systeme. In: Technikfolgenabschätzung-Theorie und Praxis 22 (2): 11-19.

Felt, Ulrike/Fouché, Rayvon/Miller, Clark A./Smith-Doerr, Laurel (Hg.) (2016): The Handbook of Science and Technology Studies (4. Auflage), Cambridge, Massachusetts: MIT Press (doi: 10.1016/S0048-7333(02)00062-8).

Geels, Frank W. (2002): Technological Transitions as Evolutionary Reconfiguration Processes: A Multi-level Perspective and a Case-study. In: Research Policy 31 (8-9): 12571274 (doi: 10.1016/S0048-7333(02)00062-8).

Geels, Frank W. (2005): Processes and Patterns in Transitions and System Innovations: Refining the Co-evolutionary Multi-level Perspective. In: Technological Forecasting and Social Change 72 (6): 681-696.

Geels, Frank W. (2011): The Multi-level Perspective on Sustainability Transitions: Responses to Seven Criticisms. In: Environmental Innovation and Societal Transitions 1 (1): 24-40.

Geels, Frank/Kemp, René (2007): Dynamics in Socio-technical Systems: Typology of Change Processes and Contrasting Case Studies. In: Technology in Society 29 (4): 441455 (doi: 10.1016/j.techsoc.2007.08.009).

Grunwald, Armin (2002): Technikfolgenabschätzung - eine Einführung, Berlin: Edition Sigma.

Hackett, Edward J./Amsterdamska, Olga/Lynch, Michael. E./Wajcman, Judy (Hg.) (2007): The Handbook of Science and Technology Studies (3. Auflage), Cambridge, Massachusetts: MIT Press.

Hocke, Peter (2016): Technik oder Gesellschaft? Atommüll als sozio-technische Herausforderung begreifen. In: Brunnengräber, Achim (Hg.): Problemfalle Endlager. Gesellschaftliche Herausforderungen im Umgang mit Atommüll. Baden-Baden: edition sigma in der Nomos Verlagsgesellschaft, 77-96 (doi: 10.5771/9783845278131-76).

Hocke, Peter/Bechthold, Elske/Kuppler, Sophie (Hg.) (2016): Rückholung der Nuklearabfälle aus dem früheren Forschungsbergwerk Asse II. Dokumentation einer Vortragsreihe am Institut für Technikfolgenabschätzung und Systemanalyse (ITAS) 
(mit Beiträgen von Detlev Möller, Beate Kallenbach-Herbert, Silvia Stumpf, Volker Metz). Karlsruhe: KIT Scientific Working Papers Nr. 47.

Hughes, Thomas P. (1983): Networks of Power: Electrification in Western Society, 18801930, Baltimore: John Hopkins University Press.

Hughes, Thomas P. (1986): The Seamless Web: Technology, Science, Etcetera, Etcetera. In: Social Studies of Science 16 (2): 281-292.

Hughes, Thomas P. (1987): The Evolution of Large Technological Systems. In: Bijker, Wiebe E./Hughes Thomas P./Pinch Trevor: The Social Construction of Technological Systems. New Directions in the Sociology and History of Technology. Cambridge, Massachusetts: MIT Press, 51-82.

Kallenbach-Herbert, Beate/Akinsara-Minhans, Anne/Brohmann Bettina/Kuppler, Sophie/Hocke, Peter/Bechthold, Elske/Brunnengräber, Achim/Isidoro Losada, Anna Maria/Themann, Dörte (2018/2019): Konzepte und Maßnahmen zum Umgang mit soziotechnischen Herausforderungen bei der Entsorgung radioaktiver Abfälle (SOTEC-radio). Arbeitsbericht zum Arbeitspaket 1. Spezifizierung der soziotechnischen Herausforderungen. Darmstadt, Karlsruhe, Berlin.

Kommission Lagerung hoch radioaktiver Abfallstoffe (2016): Abschlussbericht: Verantwortung für die Zukunft. Ein faires und transparentes Verfahren für die Auswahl eines nationalen Endlagerstandortes. K-Drs. 268, Geschäftsstelle, Berlin.

Lösch, Andreas (2012): Techniksoziologie. In: Maasen, Sabine/Kaiser, Mario/Reinhart, Martin/Sutter, Barbara (Hg.): Handbuch Wissenschaftssoziologie. Wiesbaden: Springer VS, 251-264.

Lösch, Andreas/Schneider, Christoph (2016): Transforming Power/Knowledge Apparatuses: The Smart Grid in the German Energy Transition. In: Innovation: The European Journal of Social Science Research 29 (3): 262-284 (doi: 10.1080/13511610.2016.1154783).

Pinch, Trevor J./Bijker, Wiebe E. (1987): The Social Construction of Facts and Artefacts: Or How the Sociology of Science and the Sociology of Technology Might Benefit Each Other. In: Bijker, Wiebe E./Hughes Thomas P./Pinch Trevor J.: The Social Construction of Technological Systems. New Directions in the Sociology and History of Technology. Cambridge, Massachusetts: MIT Press, 17-50.

Rammert, Werner. (2000): Kultur - Innovation - Virtualität. Technik aus soziologischer Perspektive (2. Auflage), Wiesbaden: Westdeutscher Verlag.

Rip, Arie./Kemp, René (1998): Technological Change. In: Rayner, Steve/Malone, Elizabeth (Hg.): Human Choice and Climate Change. Vol. II, Resources and Technology. Columbus, Ohio: Battelle Press, 327-399.

Roggema, Rob/Vermeend, Tim/van den Dobbelsteen, Andy (2012): Incremental Change, Transition or Transformation? Optimising Change Pathways for Climate Adaptation in Spatial Planning. In: Sustainability 2012 (4): 2525-2549 (doi: 10.3390/su4102525).

Roose, Jochen (2010): Der endlose Streit um die Atomenergie. Konfliktsoziologische Untersuchung einer dauerhaften Auseinandersetzung. In: Feindt, Peter H./Saretzki, Thomas (Hg.): Umwelt- und Technikkonflikte. Wiesbaden: VS Verlag für Sozialwissenschaften, 79-103 (doi: 10.1007/978-3-531-92354-3_4).

Smeddinck, Ulrich (2019): Sanfte Regulierung: Ressourcen der Konfliktlösung im Standortauswahlverfahren für ein Endlager. In: Deutsches Verwaltungsblatt 134 (12): 744-51. 
Weyer, Johannes (2008): Techniksoziologie. Genese, Gestaltung und Steuerung soziotechnischer Systeme, Weinheim, München: Juventa.

Weyer, Johannes/Kirchner, Ulrich/Riedl Lars/Schmidt, Johannes F. K. (1997): Technik, die Gesellschaft schafft. Soziale Netzwerke als Ort der Technikgenese, Berlin: edition sigma. 
